\title{
SoC for COFDM Wireless Communications: Challenges and Opportunities
}

\author{
Chen-Yi Lee, Hsuan-Yu Liu, and Chien-Ching Lin \\ Department of Electronics Engineering, National Chiao Tung University \\ 1001, Ta Hsueh Road, Hsinchu 300, Taiwan, ROC \\ E-mail: cylee@si2lab.org
}

\begin{abstract}
Coded Orthogonal Frequency Division Multiplexing (COFDM) technology has been widely accepted in many communication systems due to both bandwidth efficiency and robustness to channel distortion. This opens a great of opportunities for SoC society to deal with design complexity by exploiting benefits of giga-scale integration. In this paper, we'll first review the general design concept of COFDM systems and then highlight several key issues in $\mathrm{SoC}$ realization. Then a system-level design flow by taking into account both performance indices and hardware complexity will be introduced. Several core modules related to COFDM system will also be addressed to see how better solutions can be achieved, especially for wireless applications. Finally two case studies on WLAN and OFDM-UWB will be discussed to demonstrate our proposals as well as to provide some directions for further research.
\end{abstract}

\section{INTRODUCTION}

Coded orthogonal frequency division multiplexing (COFDM) technique is widely used for high-speed wireless systems such as wireless local area network (WLAN), digital video broadcasting (DVB), and ultra-wide band (UWB) system [1]-[4]. It provides high data rate and high channel capacity; for example $54 \mathrm{Mb} / \mathrm{s}$ and $480 \mathrm{Mb} / \mathrm{s}$ data rate can be respectively delivered in $20 \mathrm{MHz}-$ bandwidth IEEE 802.11a WLAN and 528MHz-bandwidth UWB. In $54 \mathrm{Mb} / \mathrm{s}$ WLAN system, BPSK/QPSK/16-QAM/64-QAM constellations are supported and so the wide range of SNR condition and the synchronization sensitivity of 64-QAM are the most focused due to the requirement of high-complexity synchronizer and channel equalizer [1]-[2]. As data rate migrates to $480 \mathrm{Mb} / \mathrm{s} \mathrm{UWB}$ [5], both wide bandwidth and supported QPSK constellation make the system less sensitive to synchronization error. To further enhance system performance, efficient error-control codes are often employed. Convolutional codes and their concatenation with Reed-Solomon codes that have been exploited in communication systems provide a superior error correction capacity while keeping a reasonable coding complexity. Low-density parity-check (LDPC) codes and Convolutional codes, which can reach a capacity approaching performance by iterative decoding algorithm, are undoubtedly the most popular research topics recently. However, the increasing demand of high speed data transmission causes the difficulty in decoder circuit design while considering low power and low cost constraints. Therefore, according to the target systems, we consider different solutions to achieve better performance through systemlevel optimization and key modules as well. With these solutions, the COFDM baseband transceiver can have opportunities to overcome the challenges of high throughput and low power under system performance requirements.

This work was supported by the National Science Council of Taiwan, R.O.C. under Grant NSC94-2215-E-009-044, and by the Ministry of Economic Affairs of Taiwan, R.O.C. under Grant 93-EC-17A-03-S1-0005.
In this paper we propose a low-complexity design concept for COFDM-based wideband system. First the advantage of wideband system containing higher tolerance to carrier frequency offset (CFO) estimation error is introduced, and hence the synchronizer complexity can be efficiently reduced based on performance consideration. Second the complexity reduction of QPSK-COFDM system is presented, the reduced SNR range and phase-processing can save computations in both symbol timing detection (STD) and channel equalization, leading to a low-cost and acceptable system solution. For low power consideration, we suggest an adaptive decoder based on channel conditions, and many redundant operations can be reduced. To achieve high-throughput, the parallel decoder architectures that are quite area efficient are presented for Viterbi decoders and LDPC decoders. Based on the proposed lowcomplexity scheme, the power efficiency of COFDM chips, defined as power per $\mathrm{Mb} / \mathrm{s}$, can be saved to below $1.2 \sim 2.3 \mathrm{~mW}$ per $\mathrm{Mb} / \mathrm{s}$ in $0.18 \mu \mathrm{m}$ CMOS process.

The paper is organized as follows. System block diagram of the proposed COFDM SOC are described in section 2. The complexitysaving schemes are described in section 3 . System performance and chip summary are respectively shown in sections 4 and 5 , followed by the conclusion in section 6 .

\section{System Block DiAgram}

FIGURE 1 shows the block diagram of the proposed COFDM baseband transceiver. The upper blocks are transmitter modules to generate the COFDM signal to satisfy the system specification, and the lower blocks are receiver modules to recover the signal and solve the channel distortion. Note that IFFT and FFT can be shared by transmitter and receiver in half-duplex mode. In the receiver, the synchronizer detects the symbol timing and CFO with the signal correlation results. Moreover, the channel equalizer solves the channel frequency response (CFR) and phase error caused by CFO estimation error and sampling clock offset (SCO) with the pilot phase error tracking (PET). The computations of conventional synchronizer and channel equalizer are done with complex divisions and multiplications which dominate the baseband processor complexity. In channel codec, the design is very critical in bit error rate (BER) performance, and channel decoding algorithm is quite computational intensive, especially those approaching Shannon limit. The large amounts of probabilistic message passing also cause enormous memory accesses. In WLAN applications, the low power Viterbi decoder [6] is applied and at most $75 \%$ memory accesses in survivor memory can be eliminated. For high speed requirement [5], a high-radix ACS structure [7] is investigated to achieve over $1 \mathrm{~Gb} / \mathrm{s}$ data throughput. On the other hand, a high performance LDPC codec $[4],[8]$ provides better improvement in BER performance and throughput. 


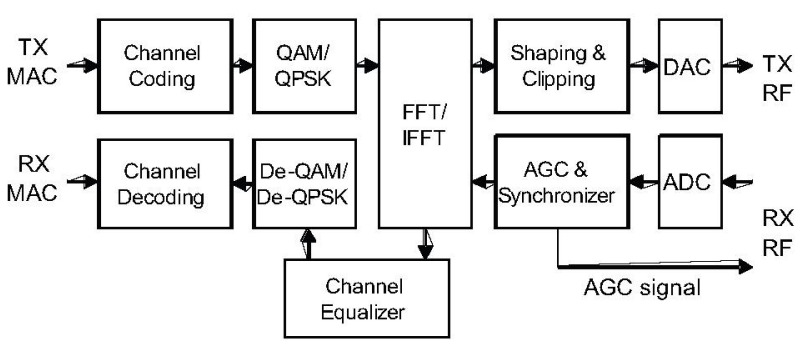

Figure 1. System BLOCK DIAGRAM OF COFDM BASEBAND TRANSCEIVER

\section{KeY COFDM TECHNIQUES}

Before addressing the design issues of each module, let's look at the system simulation platform as shown in FIGURE 2. System behavior is constructed by Matlab and $\mathrm{C}$ descriptions. Once algorithms are confirmed to system requirements, fixed-point model is set up to provide a link to hardware design. Here both IP and inhouse hardware modules can be designed and simulated at HDL level. Finally physical design can be done. With this design platform, it's easy to justify overall system performance and sub-block functionality.

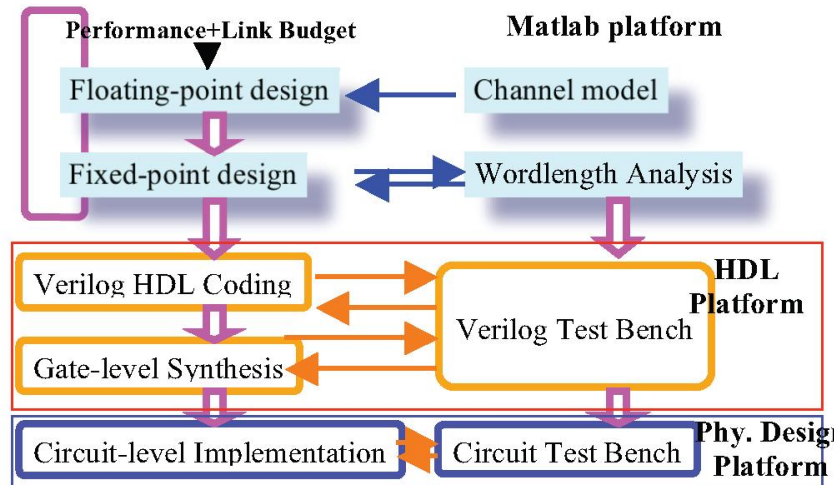

Figure 2. Proposed System-Level InTEgration Design Flow

\subsection{Low-Complexity CFO Estimation for Wideband System}

In COFDM system the signal is seriously distorted by intercarrier interference (ICI) caused by $\mathrm{CFO}$, and hence the accurate CFO estimation is required and increases the complexity of synchronizer. However the wider bandwidth can extend the subcarrier spacing to make higher tolerance to CFO estimation error. In $480 \mathrm{Mb} / \mathrm{s}$ UWB system, the subcarrier spacing is $4.125 \mathrm{MHz}$, equal to $13.2 \mathrm{x}$ of that in WLAN. According to Moose's analysis, ICI power is approximately linear to $\mathrm{CFO} /$ subcarrier spacing, and the signal-to-ICI ratio (SIR) of UWB is higher than that of WLAN by $13 \mathrm{~dB}$. In order to understand the system sensitivity to CFO which causes ICI in COFDM systems, the SNR loss for $8 \%$ packet error rate (PER) caused by CFO estimation error is drawn in FIGURE 3.

From FIGURE 3, it's found the tolerant CFO-estimation error of UWB can be higher than that of WLAN in the same SNR-loss constraint. The SNR-loss curves explain the CFO estimation of wider-band system is allowed to be more error tolerant, and the required design complexity can be reduced. Based on this wideband feature, a low-complexity synchronizer such as data-partitioned- based scheme can be used in UWB system [7], where $75 \%$ complex multiplications can be reduced to save $37 \mathrm{~mW}$ power dissipation with only $0.07 \mathrm{~dB}$ SNR loss.

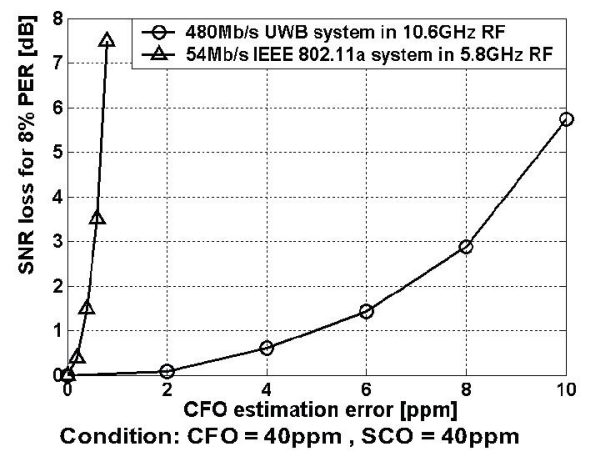

FIGURE 3. SNR LOSS FOR $8 \%$ PER CAUSED BY CFO ESTIMATION ERROR

\subsection{Low-Complexity Schemes for QPSK-COFDM System}

In WLAN system with BPSK/QPSK/16-QAM/64-QAM, the design SNR for typical $10 \%$ PER ranges in $2.5 \mathrm{~dB} \sim 26.7 \mathrm{~dB}$ [1], and while in UWB system with only QPSK constellation, the lower bound of SNR range can be increased to about $5 \mathrm{~dB}$ [4]. In synchronizer, the symbol timing is detected to avoid frame error causing high PER, and hence the frame error rate (FER) should be lower than PER for performance requirement. Compared with WLAN system, the UWB system with higher design SNR can allow higher FER, and hence the complexity of STD in synchronizer can be reduced. A tap-and-register-reduction matched filter is proposed for low-complexity UWB STD, and it can reduce $75 \%$ register size to save $28.5 \mathrm{~mW}$ power with only $0.38 \mathrm{~dB}$ SNR loss [8]. The wideband and QPSK-only feature can efficiently be exploited to reduce the design complexity of synchronizer. A trade-off between synchronizer complexity and SNR loss for typical $8 \%$ PER of UWB system is drawn in FIGURE 4. We can find a synchronizer with reduced $75 \%$ complexity can achieve an acceptable SNR loss $(\sim 0.3 \mathrm{~dB})$.

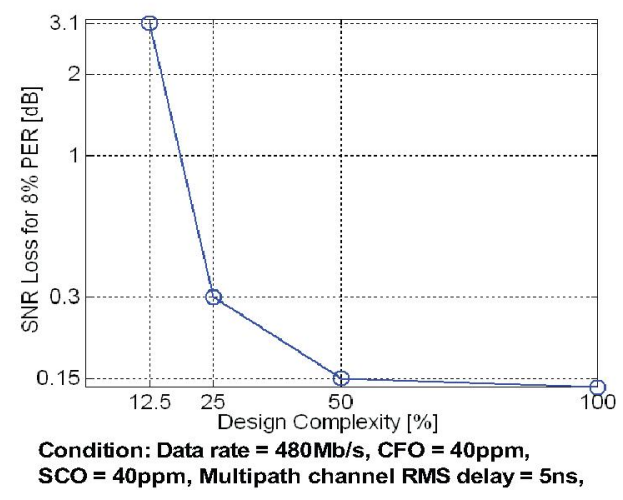

\section{FIGURE 4. TRADE-OFF OF UWB SYNCHRONIZER}

On the other hand, the QPSK constellation also implies the complexity reduction of channel equalizer. In conventional channel equalizer both divider and multiplier occupy about $60 \%$ hardware cost. Since the QPSK signal can be de-mapped with just the phase information, a divider-and-multiplier-free channel equalizer can be 
exploited [4]. In this low-complexity equalizer scheme, the phase information can be used instead of complex signals. As a result both complex divider and multiplier can be replaced by real-number adders. Furthermore the storage space storing channel information can be reduced by $50 \%$. This divider-and-multiplier-free equalizer scheme can save $61.8 \mathrm{~mW}$ power dissipation with only $0.3 \mathrm{~dB}$ SNR loss for $8 \%$ PER.

\subsection{Low-Power Viterbi Decoder for WLAN and UWB System}

Low-power Viterbi decoders for WLAN system in [1] and [6] have $30 \% \sim 40 \%$ reductions as compared to conventional designs. The modified compare-select-add (CSA) architecture lowers down hardware complexity as well as power dissipation. With the aid of path merging and prediction features, the traceback length dynamically changes according to channel conditions, and the memory accesses is reduced more than $70 \%$ on the average. Moreover, the power consumption is improved due to the reduced memory accesses. A $0.18-\mu \mathrm{m}$ test chip also shows that the power efficiency reaches $0.75 \mathrm{~mW}$ per $\mathrm{Mb} / \mathrm{s}$ at $6 \mathrm{Mb} / \mathrm{s}$ and $1.26 \mathrm{~mW}$ per $\mathrm{Mb} / \mathrm{s}$ at $54 \mathrm{Mb} / \mathrm{s}$ as specified in IEEE $802.11 \mathrm{a}$.

A high-speed Viterbi decoder is proposed in [7]. It applies the 2$\mathrm{D}$ add-compare-select (ACS) structure and the retiming mechanism. The proposed 2-D architecture provides an area efficient solution for high-radix trellis decoding, resulting in a high-level parallel decoding. Besides, the retiming techniques (RT) can be used to reduce the critical path delay of ACS for further high-speed applications. A Viterbi decoder for the UWB system [5] is implemented with the proposed ACS-4x4 approach, and experimental results indicate a $60 \%$ area reduction in contrast to radix-16 ACS based decoders. It has a considerable improvement in throughput when the retiming process is employed. Measurement of a $0.18-\mu \mathrm{m}$ test chip shows that over $500 \mathrm{Mb} / \mathrm{s}$ data rate is accomplished at $1.8 \mathrm{~V}$ supply. Furthermore, with the retiming technique, the proposed decoder can achieve $731 \mathrm{Mb} / \mathrm{s}$ data rate under the worst case comer. TABLE 1 also presents the Viterbi decoder for the UWB system [5] and highlights the effect of retiming techniques.

\section{TABLE 1. DESIGNS OF VITERBI DECODER FOR UWB SYSTEM}

\begin{tabular}{|l|c|c|}
\hline Design & ACS-4x4 & ACS-4x4 with RT \\
\hline Technology & $0.13-\mu \mathrm{m}$ & $0.13-\mu \mathrm{m}$ \\
\hline Data Throughput & $900 \mathrm{Mb} / \mathrm{s}$ & $1100 \mathrm{Mb} / \mathrm{s}$ \\
\hline Chip Area & $1.28 \mathrm{~mm}^{2}$ & $1.96 \mathrm{~mm}^{2}$ \\
\hline Gate count & $239 \mathrm{~K}$ & $358 \mathrm{~K}$ \\
\hline Chip Density & $96 \%$ & $94 \%$ \\
\hline
\end{tabular}

\subsection{High-Rate LDPC Decoder for Gb/s Wireless System}

The difficulty in realizing a parallel LDPC decoder is the signal routing congestion problem due to the randomness of paritycheck matrix and large amount of message passing. In [4], the routing issue is resolved with pipelined datapaths which have smaller signal transmission delay. In the $(1200,720)$ decoder of [8], the message memory (MMU) architecture allows parallel decoding of two codewords and minimizes the routing congestion issues with the register exchange approach. The message memory is divided into four blocks, and an additional temporal block (TMP) is included to simplify the data switch. Additionally, the data rescheduling minimizes the signal routing between datapaths and memory units as shown in FIGURE 5. Consequently, the chip becomes smaller due to the increased chip density. From simulation results, the $0.13-\mu \mathrm{m}$ chip reaches the maximum $5.92 \mathrm{~Gb} / \mathrm{s}$ data rate with $13.5 \mathrm{~mm}^{2}$ area and $268 \mathrm{~mW}$ power consumption; the chip density is about $75 \%$.

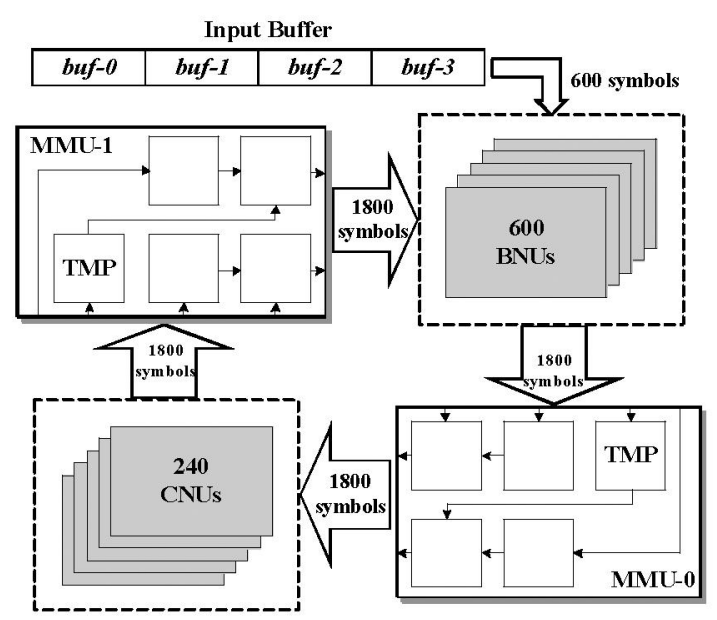

FIGURE 5. HIGH-SPEED (1200,720) LDPC DECODER

TABLE 2. SUMMARY OF $(1200,720)$ LDPC DECODER CHIPS

\begin{tabular}{|l|c|c|}
\hline Technology & $0.13-\mu \mathrm{m} 1 \mathrm{P} 8 \mathrm{M}$ & $0.18-\mu \mathrm{m} 1 \mathrm{P} 6 \mathrm{M}$ \\
\hline Data Throughput & $5.92 \mathrm{~Gb} / \mathrm{s}$ & $3.33 \mathrm{~Gb} / \mathrm{s}$ \\
\hline Power & $268 \mathrm{~mW} @ 1.2 \mathrm{~V}$ & $644 \mathrm{~mW} @ 1.8 \mathrm{~V}$ \\
\hline Chip Area & $13.5 \mathrm{~mm}^{2}$ & $25 \mathrm{~mm}^{2}$ \\
\hline Gate Count & $1.15 \mathrm{M}$ & $1.15 \mathrm{M}$ \\
\hline Chip Density & $75.4 \%$ & $71.2 \%$ \\
\hline
\end{tabular}

TABLE 2 summarizes the $(1200,720)$ LDPC decoder in $0.13-\mu \mathrm{m}$ $1 \mathrm{P} 8 \mathrm{M}$ and $0.18-\mu \mathrm{m} 1 \mathrm{P} 6 \mathrm{M}$ technologies. The data throughput is estimated with eight decoding iterations, which is sufficient for the UWB system. Note that more metal layers lead to higher chip density and better timing, but fabrication cost will be increased too.

\section{SYSTEM PERFORMANCE}

System performances of the proposed COFDM systems are listed in TABLE 3 and TABLE 4. The design SNR for typical 10\% PER of the WLAN system can be lower than the system constraint as listed in TABLE 3 [1]. When system migrates to UWB, the low-complexity schemes are used, and the transmission distances of the UWB system can be still longer than the system requirement as listed in TABLE 4. The transmission distances listed in TABLE 4 are derived from the system link-budget listed in [5] and SNR. conditions

\section{CHIP SUMMARY}

The chip micro-photo of COFDM baseband transceivers for WLAN [1] and UWB [4] are shown in FIGURE 6. From the chip summary listed in TABLE 5 , we can find the $54 \mathrm{Mb} / \mathrm{s}$ WLAN and $480 \mathrm{Mb} / \mathrm{s}$ UWB baseband chips consume $123.5 \mathrm{~mW}$ and $575 \mathrm{~mW}$, resulting in power efficiency of $2.3 \mathrm{~mW}$ and $1.2 \mathrm{~mW}$ per Mb/s. Based on the proposed low-complexity schemes, the power can be efficiently saved when system migrates from WLAN to UWB. In addition, a new version with dynamic sampling and $\mathrm{I} / \mathrm{Q}$ mismatch compensation is proposed for the $480 \mathrm{Mb} / \mathrm{s}$ multi-band (MB)-OFDM UWB chip in $0.13 \mu \mathrm{m}$ CMOS process. It consumes only $31.2 \mathrm{~mW}$ for the MODEM part, resulting in power efficiency of $0.065 \mathrm{~mW}$ per $\mathrm{Mb} / \mathrm{s}[11]$. 


\section{CONCLUSION}

In this paper, we have addressed the $\mathrm{SoC}$ design flow for COFDM wireless communications systems. At system level, a tradeoff between performance and computational complexity is done through a system simulation platform. Then all core modules are designed under target system architecture to reach better performance indices, especially in low-power issue. Research results with two test vehicles on WLAN and COFDM-UWB show that our proposal can reach better power efficiency while maintaining acceptable system transmission performance. In addition, core modules also reach better design efficiency by taking into system behavior. Currently a memory-based system architecture is explored to provide COFDMSoC solutions to solve multi-standard and multi-channel applications.

Acknowledgement: The authors would like to thank many colleagues in $\mathrm{Si} 2$ group of NCTU for many fruitful discussions in both WLAN and UWB projects. The silicon shuttle service from UMC is acknowledged as well.

TABLE 3. SNR FOR $10 \%$ PER OF COFDM-BASED WLAN SYSTEMS

\begin{tabular}{|c|c|c|c|c|c|c|c|c|}
\hline Data Rate $(\mathrm{Mb} / \mathrm{s})$ & 6 & 9 & 12 & 18 & 24 & 36 & 48 & 54 \\
\hline Design SNR (dB) & 2.5 & 4.0 & 5.2 & 7.4 & 10.1 & 14.2 & 18.6 & 20.3 \\
\hline System Constraint (dB) & 9.7 & 10.7 & 12.7 & 14.7 & 17.7 & 21.7 & 25.7 & 26.7 \\
\hline
\end{tabular}

TABLE 4. TRANSMISSION DISTANCE FOR $8 \%$ PER OF COFDM-BASED UWB SYSTEMS

\begin{tabular}{|c|c|c|c|c|c|c|}
\hline & \multicolumn{3}{|c|}{ LDPC-COFDM } & \multicolumn{3}{c|}{ MB-OFDM System } \\
System [4] & \multicolumn{3}{c|}{ [5] } \\
\hline $\begin{array}{c}\text { Data Rate } \\
\text { (Mb/s) }\end{array}$ & 120 & 240 & 480 & 110 & 200 & 480 \\
\hline $\begin{array}{c}\text { Transmission } \\
\text { Distance in AWGN } \\
\text { Channel (meters) }\end{array}$ & 14.4 & 11.5 & 8.5 & 19.1 & 13.8 & 9.0 \\
\hline $\begin{array}{c}\text { Transmission } \\
\text { Distance in } \\
\text { Multipath Channel } \\
\text { (meters) }\end{array}$ & 11.7 & 9.4 & 6.8 & 11.0 & 4.2 & 2.4 \\
\hline $\begin{array}{c}\text { System } \\
\text { Requirement } \\
\text { (meters) }\end{array}$ & 10 & 4 & 2 & 10 & 4 & 2 \\
\hline
\end{tabular}

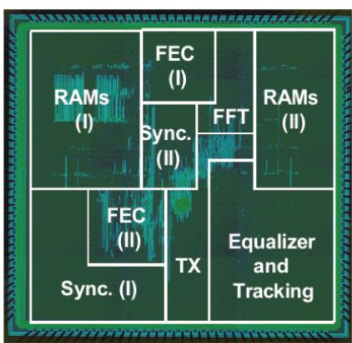

(a) COFDM -based WLAN

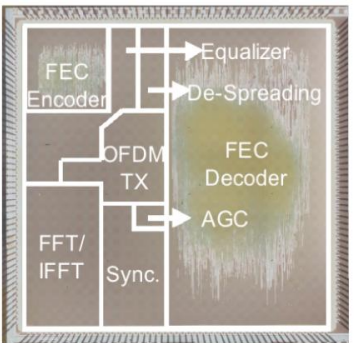

(b) COFDM -based UWB
FIGURE 6. CHIP MICROPHOTO OF COFDM BASEBAND TRANSCEIVERS

TABLE 5. CHIP SUMMARY OF COFDM BASEBAND TRANSCEIVER

\begin{tabular}{|c|c|c|}
\hline Technology & \multicolumn{2}{|c|}{$\begin{array}{c}\text { Standard } 0.18 \mu \mathrm{m} \text { CMOS, } \\
1.8 \mathrm{~V} \text { Core, 3.3V I/O }\end{array}$} \\
\hline Function & $\begin{array}{c}\text { WLAN Baseband } \\
\text { Transceiver }\end{array}$ & $\begin{array}{c}\text { UWB Baseband } \\
\text { Transceiver }\end{array}$ \\
\hline Die Size & $4.8 \times 4.8 \mathrm{~mm}^{2}$ & $6.5 \times 6.5 \mathrm{~mm}^{2}$ \\
\hline $\begin{array}{c}\text { Equivalent } \\
\text { Gate-Count }\end{array}$ & $525 \mathrm{~K}$ & $1065 \mathrm{~K}$ \\
\hline $\begin{array}{c}\text { Maximum Data Rate } \\
\text { (Mb/s) }\end{array}$ & 54 & 480 \\
\hline $\begin{array}{c}\text { RX Core Power } \\
\text { (mW) }\end{array}$ & 123.5 & 575 \\
\hline $\begin{array}{c}\text { Power Efficiency } \\
\text { (mW per Mb/s) }\end{array}$ & 2.3 & 1.2 \\
\hline
\end{tabular}

\section{REFERENCES}

[1] Hsuan-Yu Liu, Yi-Hsin Yu, Chien-Ching Lin, Ching-Che Chung, TerngYi Hsu, and Chen-Yi Lee, "A COFDM Baseband Processor with Robust Synchronization for High-Speed WLAN Applications," IEEE Symposium on VLSI Circuits, pp. 156-159, June 2004.

[2] Wolfgang Eberle, et al., "A Digital 72Mb/s 64-QAM OFDM Transceiver for $5 \mathrm{GHz}$ Wireless LAN in 0.18 um CMOS," IEEE International SolidState Circuits Conference Digest of Technical Papers, pp: 336 -337, 462463, Feb. 2001.

[3] Lei-Fone Chen, et al., "A 1.8V 250mw COFDM Baseband Receiver for DVB-T/H Application," to appear in IEEE International Solid-State Circuits Conference, Feb. 2006.

[4] Hsuan-Yu Liu, et al., "A 480Mb/s LDPC-COFDM-Based UWB Baseband Transceiver," IEEE International Solid-State Circuits Conference Digest of Technical papers, PP. 444-446, Feb. 2005.

[5] A. Batra, et al., "Multi-band OFDM Physical Layer Proposal for IEEE 802.15 Task Group 3a," IEEE P802.15-04/0943r1-TG3a, Sept. 2004 Available: http://grouper.iece.org/groups/802/15/pub/2004/15-04-049300-003a-multi-band-ofdm-cfp-document-update.zip

[6] C. C. Lin, Y. H. Shih, H. C. Chang, and C. Y. Lee, "Design of a power reduction Viterbi decoder for WLAN applications," IEEE Trans. Circuit and Syst. I, vol. 52, no. 6, pp. 1148.1156, June 2005.

[7] C. L. Chen, C. C. Lin, H. C. Chang, and C. Y. Lee, "High-Speed Viterbi Decoder Based on the Two-Dimensional ACS Structure," submitted to IEEE Trans. Circuit and Syst. I.

[8] C. C. Lin, K. L. Lin, H. C. Chang, and C. Y. Lee, "A $3.33 \mathrm{~Gb} / \mathrm{s}(1200,720)$ low-density parity-check code decoder," in proc. 31st Eur. Solid State Circuits Conf. (ESSCIRC), 2005, Sept. 2005, pp. 211-214.

[9] Lin-Hung Chen, Wei-Che Chang, Wan-Chun Liao, Hsuan-Yu Liu, and Chen-Yi Lee, "A 528MS/s Frequency Synchronizer for OFDM-Based UWB System" IEEE International Symposium on VLSI Design, Automation and Test, pp.88-91, Apr. 2005.

[10] Wei-Che Chang, Lin-Hung Chen, Wan-Chun Liao, Hsuan-Yu Liu, and Chen-Yi Lee, "An Area and Power Efficient Frame Synchronizer for $480 \mathrm{Mb} / \mathrm{s}$ OFDM-Based UWB System" IEEE International Symposium on VLSI Design, Automation and Test, pp.84-84, Apr. 2005

[11] Jui-Yuan Yu et al., "A 31.2mW UWB Baseband Transceiver with AllDigital I/Q-mismatch Calibration and Dynamic Sampling," submitted to IEEE Symposium on VLSI Circuits, June 2006. 